\title{
Decision-making in Polydrug Amphetamine-type Stimulant Users: an fMRI Study
}

\author{
Philip Koester', Kirsten G Volz², Marc Tittgemeyer ${ }^{3}$, Daniel Wagner', Benjamin Becker', \\ Euphrosyne Gouzoulis-Mayfrank' and Joerg Daumann*,' \\ 'Department of Psychiatry and Psychotherapy, University Hospital of Cologne, Cologne, Germany; ${ }^{2}$ Werner Reichardt Centre for Integrative \\ Neuroscience, Tübingen, Germany; ${ }^{3}$ Max-Planck-Institut für neurologische Forschung, Cologne, Germany
}

\begin{abstract}
Qualitative poor decision-making and associated altered neuronal activation patterns have been described for the users of several drugs, amongst others for stimulants like amphetamine and MDMA. Deficits in decision-making might be caused by an augmented attraction to short-term rewarding properties despite negative long-term consequences, leading to rigid stimulus-response patterns. In the present imaging study, we investigated decision-making and associated neuronal activation in three groups differing in their exposure to amphetamine and MDMA. An established paradigm on risky choices was used to evaluate decision-making performance and corresponding functional magnet resonance imaging ( $(\mathrm{MR}$ R) activation. Subjects could choose between a low-risk control gamble and an experimental gamble, which always differed in the probability of winning or losing, as well as the magnitudes of monetary gain or loss. Experienced users (EU), users with low exposure to stimulants and drug-naive controls, did not differ from each other in behavioral performance. In accordance with our hypotheses, the anticipation of reward led to an activation of primarily the frontal cortex and the striatum in low-exposure users and drug-naive controls. In contrast, frontal and parietal activation was observed in all groups when the actual outcome of an experimental gamble was presented. EU displayed more activation compared to both control groups when there was a high probability of winning. The study at hand supports the hypothesis that neuronal activation patterns might even differ between drug users and healthy controls when no behavioral deficits are apparent. In EU, the probability of the occurrence of an event has more influence on neuronal activation than on the actual magnitude of reinforcing properties of this event.

Neuropsychopharmacology (2013) 38, I377-1386; doi:I0.1038/npp.2013.43; published online 20 March 2013
\end{abstract}

Keywords: amphetamine; MDMA; functional magnetic resonance imaging; decision-making; reward processing; addiction

\section{INTRODUCTION}

MDMA and amphetamine are illicit substances that are popular for recreational use among young adults. These substances, together with methamphetamine, are usually referred to as amphetamine-type stimulants (ATS) (UNODC, 2011). Although the psychopharmacological properties differ substantially between the substances of this class, they are pooled together for their similarities in psychotropic effects and purpose of recreational use. Owing to their stimulating psychological effects, ATS are mainly associated with the club scene. The concomitant use of other drugs, primarily other stimulants, alcohol, and cannabis, is common in this group. Together with environmental factors such as hyperthermia and dehydration, ATS are assumed to exhibit neurotoxic properties even in recreational users (Cowan et al, 2003; Daumann et al, 2011; Kish et al, 2010; Koester et al, 2012).

\footnotetext{
* Correspondence: Professor J Daumann, Department of Psychiatry and Psychotherapy, University of Cologne, Kerpener Strasse 62, Cologne 50924, Germany. Tel: + 49221478 87113, Fax: + 49221478 97543, E-mail: joerg.daumann@uk-koeln.de Received 9 November 2012; revised 28 January 2013; accepted 30 January 2013; accepted article preview online 7 February 2013
}

Abnormalities in ATS polydrug users have been described by structural and functional magnet resonance imaging (MRI), as well as psychopathological and neuropsychological measures (Cowan et al, 2003; Daumann et al, 2011; De Win et al, 2008; Kish et al, 2010; Koester et al, 2012; Moeller et al, 2007). Besides the deficits in memory-related parameters (Becker et al, 2012; Daumann et al, 2005; Murphy et al, 2012; Wagner et al, 2012) as an assumed result of hippocampal damage, research on neurocognitive deficits associated with ATS polydrug use is mainly focused on poor decision-making (Aron and Paulus, 2007; Quednow et al, 2007). These substances exhibit strong reinforcing properties, which, for some recreational users, outweigh the well-known negative effects of substance use on psychological wellbeing and cognitive functions. The hypothesis stands to reason that poor decision-making, compulsive drug ingestion, and ultimately the development of addiction are the results of an overly attraction to shortterm rewarding properties despite negative long-term consequences (Paulus et al, 2003; Schoenbaum et al, 2006; Smerdon and Francis, 2011). This holds not only for a model of initiation of drug use, but has also been demonstrated in neuropsychological investigations (Quednow et al, 2007). 
There is little doubt about fronto-striatal circuits having a pivotal role in pitting the value of potential wins and losses against their probability of occurrence (Studer et al, 2012). The evaluation of the risk associated with a certain value of an anticipated reward has been found to be impaired in patients with damage to the orbitofrontal cortex and addicted patients (Rogers et al, 1999). Moreover, psychiatric patients and recreational as well as addicted polydrug users have been found to display an attenuated activation of the nucleus accumbens and mediofrontal regions while expecting a monetary reward (Figee et al, 2011; van Hell et al, 2010). Furthermore, ATS users have been found to make impulsive decisions when a large reward is presented, even though this reward is associated with a high risk. Although MDMA polydrug users learned the reward-contingencies, they made more disadvantageous choices compared with drug-naive controls on the Iowa Gambling Task (IGT) (Hanson et al, 2008; Moeller et al, 2007; Quednow et al, 2007; Schilt et al, 2009).

These effects seem not to be exclusively associated with ATS use, but have also been described in cocaine (Bolla et al, 2003) and cannabis (Bolla et al, 2005) users. Paulus et al $(2003,2005)$ have also demonstrated that this performance deficit is accompanied by attenuated activation of the orbitofrontal cortex, the anterior cingulate, and the parietal cortex in methamphetamine-addicted patients. The question remains whether qualitative poor decisionmaking is primarily associated with an increased sensitivity to reward or with a decreased sensitivity to punishment. Some studies suggest that both reward sensitivity and diminished responses to threatening consequences may cause disinhibited and dysfunctional choices and are, ultimately, a risk factor for the initiation and adherence of drug use (Bedi et al, 2009; Smerdon and Francis, 2011).

In the present study, we wanted to investigate decisionmaking processes in three groups of subjects who differ in the amount of ATS they use. To do so, we used an established fMRI paradigm to measure the impact of varying magnitudes and probabilities of an anticipated monetary gain or loss on the blood-oxygen level-dependent (BOLD) signal (Rogers et al, 2003, 2004). Compared with the other decision-making tasks, the task at hand had the advantage to evaluate decision-making processes apart from learning capabilities, excluding the possibility of memoryrelated confounders.

This decision-making task was applied to a group of experienced users (EU), a group with low exposure to ATS and drug-naive controls. The inclusion of a group with little exposure to these substances was a unique feature of the study at hand. On one hand, it enabled us to better control for confounding variables such as concomitant cannabis use,whereas on the other hand, it potentially revealed hints toward the answer to the question whether the findings reported for ATS users are rather a cause or consequence for drug use. Based on the literature, it was hypothesized that EU take more risky decisions compared with lowexposure users (LEU) and drug-naive controls. Furthermore, we expected that striatal and frontal areas are activated during the decision phase in all groups, but that EU display an attenuated BOLD-signal during high-risk decisions compared to LEU and drug-naive controls in mediofrontal areas.

\section{MATERIALS AND METHODS}

\section{Subjects}

Fifteen drug-naive controls (C), eighteen LEU, and fifteen EU were enrolled in the study. All participants had to be at least 18 years old. Drug-naive controls had no experience with illicit substances. Low exposure to ATS was defined as up to five pills of MDMA and/or $5 \mathrm{~g}$ of amphetamine lifetime consumption. EU were included if they at least consumed 100 doses of MDMA and/or $50 \mathrm{~g}$ of amphetamine in their lives. Overall dosages of substances used were approximated, using a dosage of $200 \mathrm{mg}$ amphetamine as a reference for a single dose. The use of methamphetamine was absent in the present sample. All participants used MDMA in the form of pills orally, or ingested amphetamine as powder nasally. Owing to the frequent concomitant use of marijuana in the sample, the use of cannabis was allowed for the two ATS-experienced groups until one day prior to imaging. On the study day, urine samples were screened for amphetamines, methamphetamines, cocaine, cannabis, benzodiazepine, barbiturates, and opiates (enzyme-multiplied immunoassay, von Minden $\mathrm{GmbH}$ ). All participants were required to stay abstinent from any legal or illegal psychotropic substance or medication 7 days prior to imaging. Randomly taken hair samples by the Institute of Legal Medicine of the University of Cologne confirmed selfreported quantity of substance use in the preceding year by comparing hair samples to drug use questionnaires.

The presences of psychiatric and neurological disorders, pathological abnormalities, as well as all contraindications to MRI were exclusion criteria. Furthermore, participants were not allowed a positive drug screen on the day of the study with the exception of cannabis. Subjects with current or previous alcohol abuse or dependence (according to DSM IV criteria), and subjects with a regular use of other illicit drugs (regular was defined as use once a month or more often over 6 months or longer over the past 2 years) were not included in the study. Following a detailed description about the study, written informed consent was obtained from all participants. Subjects had the assurance that they could withdraw from the study at any time without having to explain the reasons and received a payment for their participation. The present study was approved by the local ethics committee and is in accordance with the declaration of Helsinki.

\section{Statistical Analysis of Demographic Characteristics and Behavioral Measures}

All demographic and behavioral data were analyzed using IBM SPSS Statistics 19. Demographic sample characteristics and drug use patterns were analyzed by means of analysis of variance (ANOVA), Mann-Whitney $U$-test, and $\chi^{2}$ test. The analysis of task-related behavioral measures was performed with repeated measures ANOVAs. The proportion of trials on which the participants chose the experimental gamble over the control gamble was computed for each condition. An arcsine transformation was applied to each condition of the proportionate choices for the experimental gambles, which is appropriate whenever the variance of a measure is proportional to the mean. Using this method, trials in which the participant did choose the control gamble added to the 
mean as a zero, thereby alleviating the problem of missing values in the repeated measures' analysis. These arcsinetransformed proportions served as dependent variables. A repeated measures ANOVA was applied to these variables with group (controls, LEU, and EU) as between-subject factors and conditions (probability of winning, possible gain, and possible loss) as within-subject factors.

\section{(f)MRI Image Acquisition}

Echo planar imaging (EPI) images were acquired on a 3 Tesla Siemens Magnetom Trio with a standard quadrature head coil. The parameters for 1022 functional volumes were: voxel size of $3,3 \times 3$, and $3 \times 4 \mathrm{~mm}$, TR of $2000 \mathrm{~ms}$, TE of $80 \mathrm{~ms}$, field of view (FoV) $210 \mathrm{~mm}$, a matrix size of $64^{2}$, and a flip angle of $10^{\circ}$. To improve the coregistration of functional images to the standard brain, additionally T1weighted images were obtained with parameters of: voxel size $1 \times 1 \times 1.3$, TR of $2000 \mathrm{~ms}$, TE of 5.8, and a flip angle of $18^{\circ}$. Local field inhomogenities were accounted for by means of field map reconstruction.

\section{Paradigm}

The paradigm was adopted from Rogers et al (2003, 2004). The decision-making task can be divided into two phases. In the decision-phase, subjects were shown two histograms depicting two gambles that differ in the amount and probability of winning or losing a certain amount of money (see Figure 1). One of these gambles was always a control gamble where subjects have a $50 \%$ chance of winning or losing a small amount $(10 \mathrm{ct})$ of money. For the experimental gamble, there was either a high $(75 \%)$ or low $(25 \%)$ chance of winning or losing a high $(80 \mathrm{ct})$ or low (20 ct) amount of money. Thus, in the decision-phase, the participant always chooses a gamble based on the incentive magnitude and the probability of winning. In each trial, the participant had to choose between an experimental and control gamble within $4 \mathrm{~s}$, which were presented randomly on either the left or right side of the screen. If the participant did not press any button, he was encouraged to focus the attention on the game after these $4 \mathrm{~s}$. Trials in which the participant gave no response did not enter the analysis. In the outcome-phase, a green upward arrow or red downward arrow, and the amount of credit points were displayed to indicate whether the participant won or lost the gamble, followed by a feedback on how many credit points

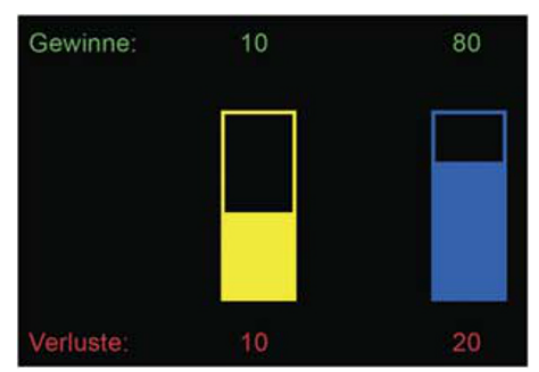

Figure I This example depicts a control gamble (left) with a $50 \%$ chance of winning ('Gewinne') or losing ('Verluste') $10 \mathrm{ct}$, and an experimental gamble (right) with a chance of $75 \%$ to win $80 \mathrm{ct}$ and a $25 \%$ chance to lose $20 \mathrm{ct}$. the participant has gathered overall. This phase lasted for $4 \mathrm{~s}$. Altogether, seven blocks, each containing 20 gambles, were presented. In each block, the subject started with 100 credit points. The task was programmed and presented with Presentation Software (Neurobehavioral Systems). Before the scanning session, participants were given a brief sequence of practice trials to assure that they understood the task. The total duration of the task was $\sim 20 \mathrm{~min}$.

\section{Statistical Analysis of fMRI Data}

The analysis of the fixed interval event-related design was carried out using FEAT 5.98, part of FSL (FMRIB Software Library) (Smith et al, 2004; Woolrich et al, 2009). First, all functional images were corrected for head motion using MCFLIRT (Jenkinson et al, 2002), fieldmap reconstruction, and slice time correction. Mean displacement must not have exceeded $1.5 \mathrm{~mm}$. Additionally, displacement graphs have been inspected visually to exclude the possibility of rapid head movements distorting the results. In the following, the skull has been removed using BET (Smith, 2002). Field inhomogenities were accounted by field map reconstruction. A coregistration of functional images and individual T1-weighted images, and the registration of this structural image to the Montreal Neurological Institute 152 (MNI 152) facilitated a valid reconstruction of specific activated brain regions. Spatial smoothing of functional images was applied with a smoothing kernel of $8 \mathrm{~mm}$ full width at half maximum (FWHM).

Time-series statistical analysis was carried out using FMRIB's Improved Linear Model (FILM) with local autocorrelation correction (Woolrich et al, 2001). Z-statistic images were thresholded using clusters determined by $Z>2.3$ and a corrected cluster significance threshold of $p=0.05$ (Worsley et al, 1992). Registration to high resolution and/or standard images was carried out using FLIRT (Jenkinson et al, 2002; Jenkinson and Smith, 2001). FMRIB's Local Analysis of Mixed Effects-1 (Flame 1) was used for modeling and estimating the inter-session random-effects component of the mixed-effects variance. Additionally, in fMRI, higher level group comparisons were modeled by means of ordinary least square (OLS), which is less accurate compared with FLAME, but also more liberal regarding the first-level variances. Correlations between first-level activation and the most important drug use parameters were calculated for low exposure and EU. Years of education was added as a covariate to group all the statistics.

Each condition was defined of either belonging to a decision-phase or outcome-phase. The decision-phase started with the presentation of the gamble and lasted for $4 \mathrm{~s}$. The outcome-phase began with the presentation of the arrow, indicating whether the subject won or lost the gamble and lasted till the presentation of an overall score of credit points. Two models were set up separately to analyze the BOLD signal for different trial types in the first level analysis. Using only one model, several trials would have been defined belonging to two different conditions simultaneously. In the first model, the effects of high $v s$ low probability of winning, positive $v s$ negative outcome, an overall measure for probability of winning $v s$ control, and the overall effect of outcome $v s$ control were analyzed for each subject. In the second model, anticipation high gain $v s$ 
low gain and high loss $v s$ low loss, the actual positive and negative feedback, as well as an overall measure of all experimental trials $v s$ all control trials were analyzed. In the following, the two models were processed separately; no inferences were made between the two analyses.

Thus, effects of a specific condition were calculated by subtracting both trial types within one model (high/low; experimental/control) belonging to this condition (eg, low probability of winning-high probability of winning for the effects of low probability and vice versa). In the following, 'neuronal activity' refers to an elevation of the BOLD signal.

\section{RESULTS}

\section{Demographic Characteristics and Drug use Patterns}

The results are summarized in Table 1. All Groups were comparable in terms of age $(p=0.089)$ and gender distribution $(p=0.907)$. However, drug-naive controls had significantly more years of education compared with LEU $(p=0.037)$ and $\mathrm{EU}(p=0.008)$. Virtually, all participants were polydrug users. Imaging group effects were not affected by differentially distributed (sub) acute effects of cannabis use, as LEU and EU did not differ with respect to a positive screening of recent cannabis use (Mann-Whitney $U=127.5 ; p=0.789$ ).

As can be seen in Table 2, both the ATS-using groups did differ from each other with respect to specific MDMA and amphetamine use patterns, but not with respect to cannabis use patterns.

\section{Behavioral Measures}

Detailed performance data are presented in Table 3. Trials in which participants did not respond did not enter the analyses. Drug-naive controls did not respond in $0.8 \%$ of the trials. LEU and EU missed 1.7 and $1.5 \%$ of the trials, respectively. This difference between the three groups was statistically not significant $((\mathrm{F}(1,45)=0.96, p=0.39))$.

Although drug-naive controls chose significantly fewer experimental gambles (mean: 444) compared with LEU (mean: 516) and EU (mean: 50,7) (F $(1,45)=3.829$, $p=0.029$ ), all groups won a comparable amount of money (F $(1,45)=0.446, p=0.643)$. All groups chose more experimental games when the probability of winning was high $(\mathrm{F}(1,45)=661.3, p>0.001)$, the possible gains were high $(\mathrm{F}(1,45)=102.0, p>0.001)$, and the possible losses were low $(F(1,45)=112.9, p>0.001)$. Groups did not differ from each other with respect to the number of decisions for one specific trial (probability: F $(1,45)=0.791, p=0.460$; possible gain: $\mathrm{F}(1,45)=1.523, p=0.229$; possible loss: $\mathrm{F}(1$, $45)=0.191, p=0.827)$. The groups were also comparable in terms of deliberation time when choosing an experimental gamble $(\mathrm{F}(1,45)=0.03, p=0.971)$. All participants reacted faster when they chose a control game compared with when they chose an experimental game (F $(1,45)=9.55$; $p=0.003$ ). However, the interaction effect of deliberation time with game type and group did not reach significance $(\mathrm{F}(1,45)=3.05, p=0.057)$.

\section{fMRI Between-Group Effects}

All relevant between-group statistics acquired using OLS are summarized in Table 4. Main findings are additionally displayed in Figure 2. Modeling the higher-level analysis with FLAME did not reveal any significant group differences between EU, LEU, and drug-naive controls. As the lack of significant group differences was most probably associated with a high variance in the first-level analyses, we decided to additionally perform a higher-level model using OLS. This analysis revealed significant group differences for two conditions. When an experimental gamble was associated with a high gain, LEU, and drug-naive controls displayed a higher BOLD signal in the lateral frontal cortex compared with EU. In contrast, when the probability of winning was high, the right parietal lobe showed more activation in experienced and LEU compared with drug-naive controls. Correlational analyses within the fMRI-design did not reveal any association between the BOLD signal, years of education, and the age of all participants. Further correlational analyses were performed with the time elapsed since

Table I Demographic Characteristics of the Investigated Sample

\begin{tabular}{|c|c|c|c|c|c|}
\hline Characteristics & Controls $(n=15)$ & Low exposure $(n=18)$ & Experienced $(n=15)$ & $\mathbf{F}^{\mathbf{a}} / \mathbf{U}^{\mathbf{b}}$ & $P$ \\
\hline Present age & $26.47( \pm 4.21)$ & $22.89( \pm 4.07)$ & $25.60( \pm 6.0)$ & $2.56^{\mathrm{a}}$ & 0.089 \\
\hline Gender (male: female) & $9: 6$ & $12: 6$ & $10: 5$ & $0.375^{b}$ & 0.829 \\
\hline Education & $17.50( \pm 2.78)$ & $15.13( \pm 2.5 \mid)$ & $14.47( \pm 2.02)$ & $5.87^{\mathrm{a}}$ & 0.006 \\
\hline \multicolumn{6}{|l|}{ Experience with } \\
\hline Cannabis & 0 & 18 & 15 & $135.000^{\mathrm{b}}$ & 1.000 \\
\hline \multicolumn{6}{|l|}{ Last use of } \\
\hline MDMA (days) & - & 930.65 (1094.607) & $32.87(44.346)$ & $20^{\mathrm{b}}$ & $<0.001$ \\
\hline
\end{tabular}

${ }^{\mathrm{a}} \mathrm{F}$-values with post-hoc test (Scheffé)

${ }^{b}$ Comparison tested with Mann-Whitney U-test 
Table 2 Specific Drug Use Patterns of Beginning and Experienced Users. t-values were Calculated Using Unpaired t-test; Two-tailed $(\mathrm{df}=48)$

\begin{tabular}{|c|c|c|c|c|c|}
\hline Specific drug use patterns & Controls $(n=15)$ & Low exposure $(n=18)$ & Experienced $(n=15)$ & $U$ & $P$ \\
\hline Lifetime dose MDMA (pills) & - & $2.65( \pm 1.47)$ & $350.66( \pm 229.89)$ & 0 & $<0.001$ \\
\hline Lifetime dose amphetamine (g) & - & $3.12(1.76)$ & $322.80( \pm 346.92)$ & 0 & $<0.00$ । \\
\hline Lifetime dose cannabis (g) & - & $793.96( \pm 986.92)$ & $10 \mid 8.87( \pm \mid 386.86)$ & 125.5 & 0.735 \\
\hline Dose MDMA (pills/occasion) & - & $1.18( \pm 0.68)$ & $3.63( \pm 2.43)$ & 23 & $<0.001$ \\
\hline Dose Amph (mg/occasion) & - & $519.44( \pm 321.36)$ & $926.67( \pm 510.56)$ & 69 & 0.016 \\
\hline
\end{tabular}

Table 3 Untransformed Number of Proportionate Choices; F and p-values are Derived After Arcsine Transformation and Refer to the Contrast of High vs Low

\begin{tabular}{|c|c|c|c|c|c|c|}
\hline & Contrast & Controls & Low exposure & Experienced & $\mathbf{F}$ & $P$ \\
\hline \multirow[t]{2}{*}{ Probability } & High vs & $0.75 \pm 0.03$ & $0.79 \pm 0.03$ & $0.76 \pm 0.03$ & 515.88 & $<0.001$ \\
\hline & low & $0.04 \pm 0.01$ & $0.12 \pm 0.01$ & $0.13 \pm 0.02$ & & \\
\hline Expected gain & Low & $0.32 \pm 0.02$ & $0.36 \pm 0.02$ & $0.37 \pm 0.02$ & & \\
\hline Expected loss & High vs & $0.28 \pm 0.03$ & $0.34 \pm 0.03$ & $0.32 \pm 0.03$ & 105.04 & $<0.001$ \\
\hline
\end{tabular}

Table 4 Specific fMRI Results. Between-Group Effects Refer to Results Obtained by Applying OLS; Within-Group Effects were Obtained by Using FLAME

\begin{tabular}{|c|c|c|c|c|c|c|c|c|c|c|}
\hline Group & Contrast & Cluster & Voxel & $p$ & $Z$ max & $\mathrm{Pe}$ & $\mathbf{x}$ & $\mathbf{Y}$ & $\mathbf{Z}$ & Location \\
\hline$C>E U$ & High-low possible gain & । & 1193 & $<0.01$ & 3.9 & 11.92 & -64 & -2 & 28 & Frontal lobe, $\mathrm{R}$ \\
\hline LEU > EU & High-low possible gain & । & 1498 & $<0.01$ & 3.98 & 18.26 & -54 & 4 & 36 & Frontal lobe, $\mathrm{R}$ \\
\hline$E U>C$ & High-low probability & I & 728 & 0.04 & 4.09 & 15.44 & -32 & -58 & 10 & Parietal lobe, $\mathrm{R}$ \\
\hline$L E U>C$ & High-low probability & I & 1009 & $<0.01$ & 3.83 & 9.56 & -38 & -42 & 6 & Parietal lobe, $\mathrm{R}$ \\
\hline C & Exp-con gamble (decision) & I & 2804 & $<0.01$ & 4.23 & 57.86 & 6 & 30 & 40 & Frontal lobe, $\mathrm{R}$ \\
\hline \multirow[t]{6}{*}{ LEU } & Exp-con gamble (decision) & I & 5284 & $<0.01$ & 4.11 & & 22 & 44 & 36 & Frontal pole, $\mathrm{R}$ \\
\hline & Exp-con gamble (decision) & 2 & 2645 & $<0.01$ & 4.37 & 32.28 & -10 & -14 & 8 & Thalamus, L \\
\hline & Exp-con gamble (decision) & 3 & $|50|$ & $<0.01$ & 3.72 & & -36 & -58 & 48 & Parietal lobe, L \\
\hline & Exp-con gamble (outcome) & I & 6029 & $<0.01$ & 4.84 & 34.31 & -10 & 58 & -4 & Frontal pole, $\mathrm{L}$ \\
\hline & Low-high probability & 4 & 896 & 0.03 & 4.36 & & 34 & 18 & 18 & Frontal lobe, $\mathrm{R}$ \\
\hline & Positive outcome & I & 3445 & $<0.01$ & 4.04 & 15.07 & 36 & -12 & 52 & Frontal lobe,R \\
\hline \multirow[t]{5}{*}{ EU } & Positive outcome & I & 3059 & $<0.01$ & 4.3 & 16.6 & -4 & -46 & 38 & Parietal lobe, L \\
\hline & Exp-con gamble (outcome) & I & 3273 & $<0.01$ & 4.11 & & 6 & 66 & 22 & Frontal pole, $\mathrm{R}$ \\
\hline & Exp-con gamble (outcome) & 2 & 1739 & $<0.01$ & 4.43 & 32.13 & 54 & -48 & 16 & Parietal lobe, $\mathrm{R}$ \\
\hline & Exp-con gamble (outcome) & 3 & 1089 & $<0.01$ & 4.58 & & 12 & -50 & 34 & Parietal lobe, $\mathrm{R}$ \\
\hline & Exp-con gamble (outcome) & 4 & 884 & 0.04 & 4.15 & & 50 & -62 & 20 & Parietal lobe, $\mathrm{L}$ \\
\hline
\end{tabular}

Abbreviations: C, control group; con, control gamble; EU, experienced users; exp, experimental gamble; L, left hemisphere; LEU, low-exposure users; Pe, parameter estimates; $R$, right hemisphere.

$\mathrm{X}, \mathrm{Y}$, and $\mathrm{Z}$ are $\mathrm{MNI} 152$ coordinates of the $Z$-peak voxel within a cluster. 


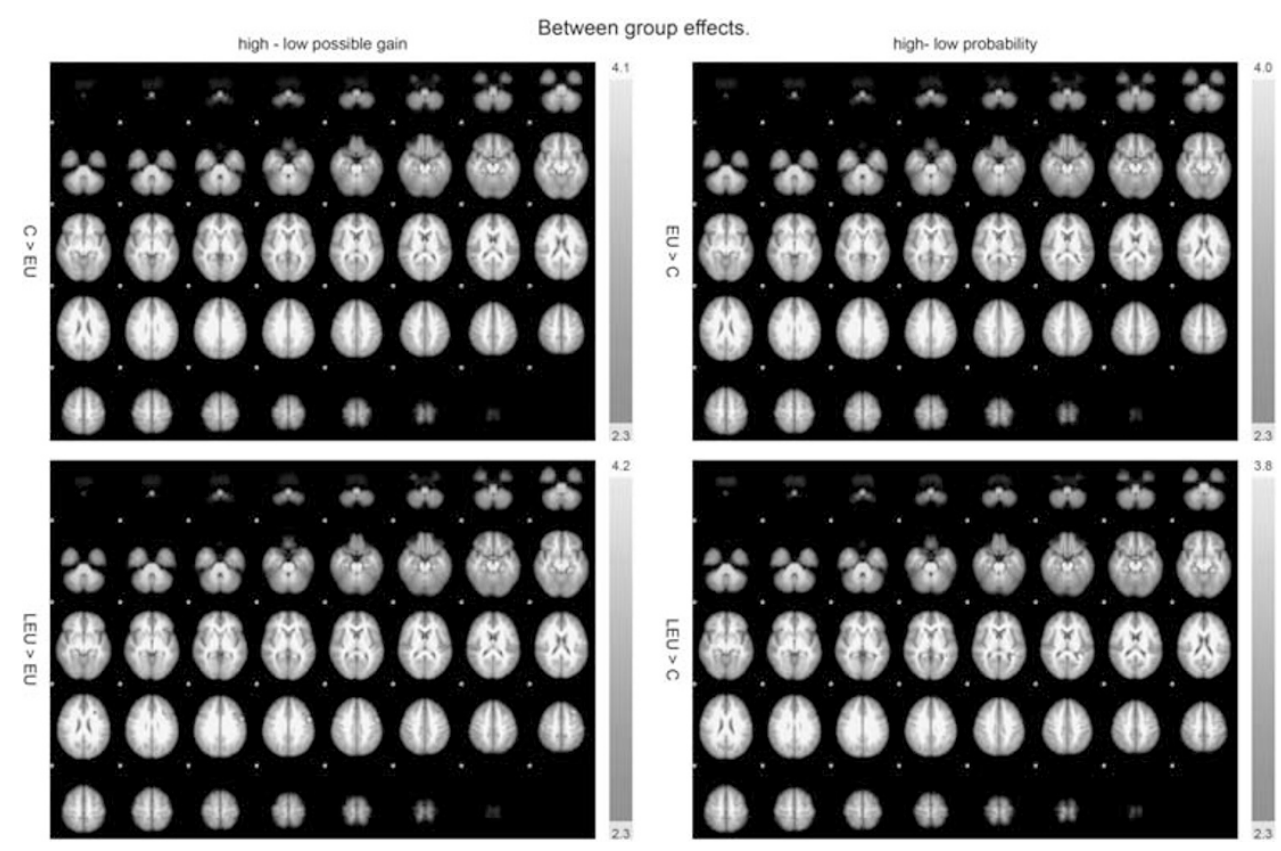

Figure $2 \mathrm{fMRI}$ between group effects corrected for multiple comparisons using OLS. The bar indicates the Z-values.

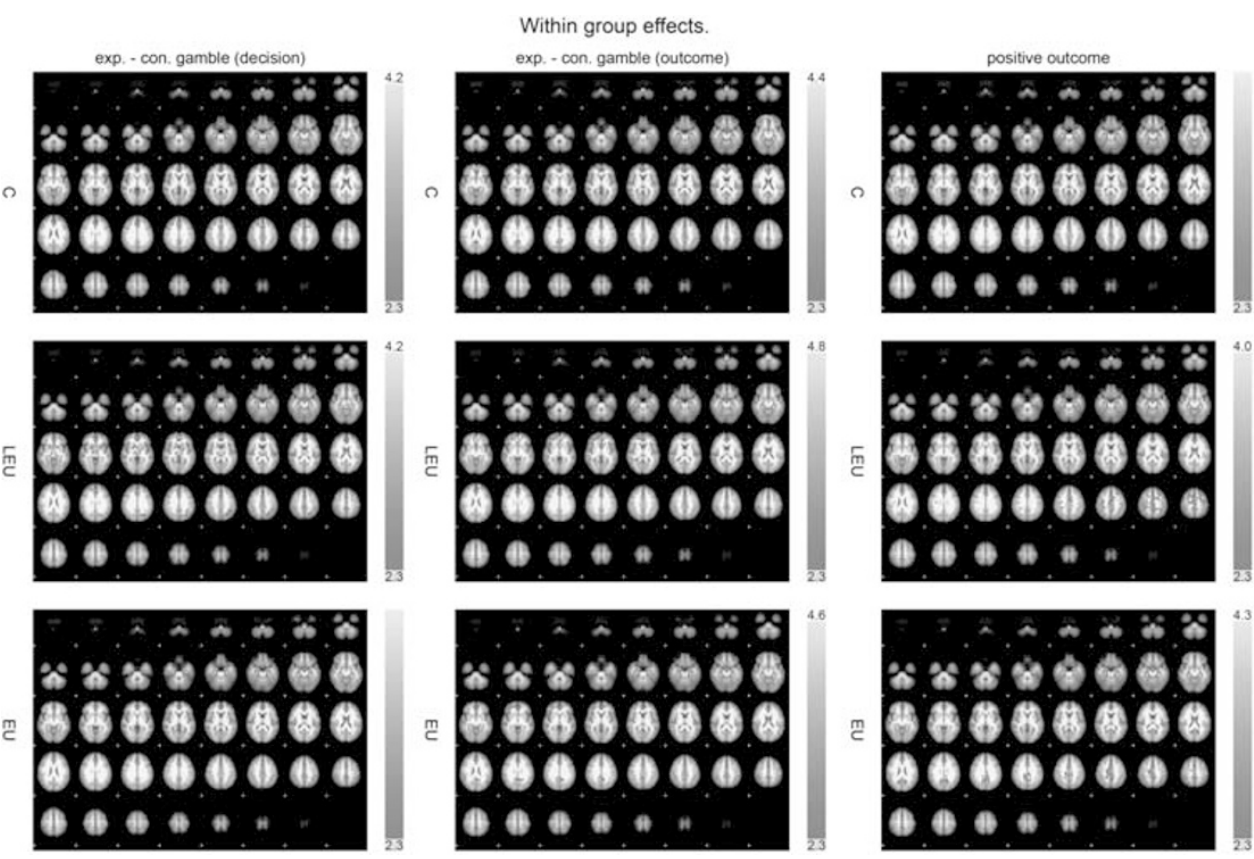

Figure 3 fMRI within group effects corrected for multiple comparisons using FALME. The bar indicates the Z-values.

last use of amphetamine, MDMA, and cannabis, as well as the average amount consumed per occasion and cumulative lifetime consumption. These analyses did not reveal any associations with the BOLD signal.

\section{fMRI Within-Group Effects}

All relevant within-group statistics acquired using FLAME are summarized in Table 4. Main findings are additionally presented in Figure 3. In the decision-phase, a superior frontal activation was observed in the ATS-using groups when participants chose an experimental over a control gamble. In the same condition, controls additionally displayed a significant increase in frontal and striatal activation. When the probability of winning the experimental gamble was low, LEU but not EU displayed an elevated BOLD signal in striatal areas, as well as the mediofrontal and parietal cortex. In the outcome-phase, all three groups displayed a significant BOLD signal of the parietal and mediofrontal cortex when the outcome of an 
experimental gamble was presented compared with when the outcome of a control gamble was presented. Winning the experimental gamble led to an activation of the parietal cortex in EU. LEU additionally displayed a medio-superior activation while receiving a win. As in the group of EU, a positive outcome of an experimental gamble was associated with a mediofrontal and parietal activation. Drug-naive controls did not display these specific activations.

\section{DISCUSSION}

The study at hand investigated behavioral performance and associated neuronal activation patterns in the two groups of ATS-polydrug users, and a control group performing a decision-making task. All groups were comparable in terms of demographic characteristics, except the years of education. As cannabis use patterns did not differ between the ATS groups, it is unlikely that (sub) acute effects of marijuana had a major influence in the result of the present study.

Drug-naive controls chose less experimental gambles compared with the ATS groups. Overall performance, assessed with the proportionate choices, deliberation times, and actual outcome, did not differ between the three groups. LEU and drug-naive controls, but not EU, displayed an expected striatal and mediofrontal activation when choosing an experimental gamble over a control gamble. Furthermore, all groups displayed a medio- and orbitofrontal activation while perceiving the outcome of their experimental gamble compared with the control gamble, irrespective of winning or losing the game. In a second, more liberal analysis, this effect was different for the three groups, with drug-naive controls and LEU showing more frontal activation compared with $\mathrm{EU}$ when the associated win of an experimental gamble was high. In contrast, when the probability of winning was high, EU and LEU displayed an elevated activation compared with drug-naive controls. Correlational analyses in the present study did not reveal any associations between specific drug use patterns, behavioral performance, and neuronal activation.

The results of the present study are, for the most part, in line with our a priori hypotheses and literature on this topic. A high overlap of our results with other reports and the fact that despite a priori hypotheses, a whole-brain analysis was applied, validate the sensitivity of the paradigm used (Figee et al, 2011; Knutson et al, 2001; Rogers et al, 1999; Tobler et al, 2009; van Hell et al, 2010). In short, the anticipation of reward primarily activated frontal and striatal regions, whereas the receipt of reward exclusively activated orbito- and mediofrontal regions. EU displayed an attenuated signal both at the anticipation and the actual receipt of a reward. These results support the hypothesis that massive ATS polydrug use is associated with functional alterations of the frontostriatal reward circuitry, whereas little ATS exposure only has a marginal effects on these functional parameters. EU did not differ from LEU and drug-naive controls in terms of behavioral performance. Thus, although functional alterations were found, these effects were not detectable in behavioral measures. This finding might be interpreted in two ways. First, functional alterations as a result of ATS use might become apparent even before qualitative poor decision-making on behavioral tests. Second, the paradigm at hand was potentially not specific enough in a way that the gap between risky choices and save choices was too big to provoke impulsive and risky decisions in recreational ATS users. Paulus et al (2003) described rigid stimulus-response patterns and a reduction of mediofrontal activation in methamphetamine subjects performing a decision-making task where there was no monetary loss possible (Paulus et al, 2003). Accordingly, the lack of behavioral differences in the present study might be caused by adaptive processes, whereby ATS users give cautious responses and thereby do not differ from the control groups.

It is difficult to interpret the results solely in terms of an overly attraction to reward or a reduced sensitivity to punishment. The attenuated frontal signal of EU despite normal performance might reflect a rigid stimulus-response pattern, which does not differ from controls on a behavioral level. In the same manner, the parietal activation of EU when the probability of winning was high might reflect an elevated attentional focus on processes corresponding to the actual availability of a reinforcer, in this case the win of the gamble. This hypothesis is supported by literature on the involvement of the parietal cortex in ATS users performing a decision-making task. It is hypothesized that the parietal cortex is associated with risk-taking behavior (Paulus et al, 2005), as well as executive and inhibitory processes associated with decision-making (Aron and Paulus, 2007; Roberts and Garavan, 2010). Therefore, the group analyses support the hypothesis that a high probability of winning a gamble recruits more neuronal activation than the actual height of a possible win in EU.

Although fMRI studies mostly demonstrate an attenuated activation of frontostriatal circuits in drug abusers performing a decision-making task, reports on qualitative deficits in behavioral performance are highly inconsistent and might therefore be associated with task- and sample characteristics. Poor decision-making has been demonstrated in ATS, opiate, and marijuana users (Bolla et al, 2005; Rogers et al, 1999; Schilt et al, 2009). However, other reports did not report any performance deficits in cocaine abusers (Bolla et al, 2003), subjects at high risk for alcoholism (Cservenka and Nagel, 2012), and MDMA users (Ersche et al, 2005), despite fMRI activation patterns comparable to the present study.

Beside a high overlap with other fMRI studies on decision-making, the results of the present study are also in line with reports on structural deficits in ATS polydrug users (Cowan et al, 2003; Daumann et al, 2011; Kish et al, 2010; Koester et al, 2012). A decrease in gray matter volume (Daumann et al, 2011) and a thinning of the cerebral cortex (Kish et al, 2010; Koester et al, 2012) have been described primarily in the orbito- and mediofrontal cortex, as well as the parietal cortex of MDMA and amphetamine users (Cowan et al, 2003). Although the exact mechanisms of neuronal degeneration are not yet fully understood, druginduced changes in synaptic strength and structure have been proposed (Hutton et al, 2009). Furthermore, cellular adaptations in dopaminergic and/or glutamatergic projections as a result of chronic drug use have been proposed to underlie alterations in OFC (Schoenbaum and Shaham, 2008; Volkow et al, 2009). Owing to this overlap of 
functional and structural imaging results, the hypothesis substantiates that alterations on a neuronal basis might even influence functional characteristics of the investigated circuits.

The reward circuitry, which, amongst other regions, encompasses the orbito- and mediofrontal cortex and the lateral striatum, has not only been identified as a core region for decision-making, but has also been ascribed a major role in models of addiction (Koob and Simon, 2009; Schoenbaum et al, 2006). The frontal cortex is important for salience attribution, and processing information on rewarding and punishing properties of stimuli (Roesch and Olson, 2004; Rolls, 2004). The reinforcing properties of ATS are mainly mediated by an elevated dopamine release in the nucleus accumbens. During the transition from recreational use to addiction, the activation of the lateral striatum diminishes and thereby reduces the importance of the reinforcing properties. Simultaneously, the prefrontal cortex has been found to become hypersensitive to the prediction of the occurrence of rewarding properties (Kalivas and Volkow, 2005). The results of the present study are in line with this model, as EU did not display NAcc activation while choosing an experimental gamble, showed an attenuated signal compared with the other groups when a high gain was anticipated and an elevated signal compared with the other groups when the probability of winning was high. Therefore, the changes of signal in this circuitry might not only account for models of addiction but might also be associated with poor decision-making of ATS users in general (Schoenbaum et al, 2006). The hypothesis of rigid stimulus-response behavior does not exclude the possibility of an altered sensitivity to reward and punishment in ATS polydrug users. Rather it might reflect different stages of addictive behavior, with an elevated sensitivity leading to drug use in the first place which, in turn, results in a rigid behavior where the reinforcing properties itself become less important than the reduction of craving symptoms (Kalivas and Volkow, 2005). Still, despite a well fit between models of addiction and poor decision-making, and a high overlap between functional and structural imaging results, these data give no information whether the findings are rather a cause or consequence of drug initiation and adherence. The same holds for the results of the present study, which either might be interpreted as a drug induced alteration of reward circuits, or as a vulnerability to drug use in the first place.

Several limitations are inherently linked to this naturalistic design of the study. First of all, we cannot exclude pre-existing group differences. Despite a thoughtful and throughout acquisition of demographic data and drug use patterns, interactive effects between the substances cannot be disentangled. In this context, all subjects in the ATS groups were polydrug users. Results of the present study therefore cannot be linked to the effects of one specific substance or interaction effects between substances. However, the aim of the investigation was to study the effects of decision-making in typical ATS users, which are commonly polydrug users. Another limitation of the present study is associated with the lack of data concerning specific alcohol and nicotine consumption patterns. Alcohol and nicotine use have repeatedly been associated with reduced gray matter volume and cortical thickness in the prefrontal cortex and the dorsal ACC (Gallinat et al, 2006; Kuhn et al, 2010; Lawyer et al, 2010; Mechtcheriakov et al, 2007). If the consumption patterns differed between the groups, this might have affected the results of the present study.

There are also limitations associated with the applied paradigm and fMRI acquisition. A significant problem with the paradigm used was the fact that the two event types, probability, and incentive magnitude, always occur simultaneously, and therefore cannot be analyzed separately. Thus, we cannot guarantee that these factors are truly separate, and future studies will need to dissociate them decisively to determine the extent of their possible colinearity. Furthermore, the high variability of activation patterns in the first-level analysis is most probably the cause of missing group statistics in the FLAME analysis and the absence of correlational results between activation patterns and drug-use characteristics. However, the imaging results of the present study are considered to be valid as statistic thresholds, smoothing and correction for multiple comparisons were chosen conservatively.

Future work might shed more light on parallels between models of addiction and decision-making under laboratory setting. Sensitive designs are needed to detect a threshold where poor performances on decision-making tasks become apparent, and to detect how these findings translate into functional alterations in the reward circuitry. A thorough acquisition of further neuropsychological data and psychopathological diagnostics should accompany research on this topic. Especially, measures on impulsivity might provide important information on decision-making differences between ATS polydrug users and drug-naive controls. Besides the evaluation of decision-making using positive reinforcement by means of monetary gain, a model implementing negative reinforcement might match better to current models of addiction. However, both suggestions are practically difficult to implement.

\section{CONCLUSION}

The study at hand supports the hypothesis that heavy ATS use is associated with altered neuronal activation patterns. A reduction of frontal and striatal, and an elevation of parietal activation were found in EU compared with LEU and drug-naive controls for different trial types. Based on the BOLD signal, the hypothesis stands to reason that EU, and probably even addicted subjects, might be primarily attracted by the availability of a reinforcer compared to the magnitude of reinforcing properties of this stimulus. Activation patterns were found to be altered in EU despite a behavioral performance comparable to LEU and drugnaive controls. The dissociation of behavioral and neuronal alterations of the frontostriatal reward circuitry might not only give valuable information regarding decision-making, but also contribute to the understanding of compulsive drug use in the first place.

\section{ACKNOWLEDGEMENTS}

This study was supported in part by a grant to E GouzoulisMayfrank and J Daumann from the Deutsche Forschungsgemeinschaft (DFG GO 717/6-1/2). We additionally would 
like to thank Hendrik Koester for the production of the images. Marc Tittgemeyer is supported by the German Research Foundation in the Clinical Research Group 219.

\section{DISCLOSURE}

The authors declare no conflict of interest.

\section{REFERENCES}

Aron JL, Paulus MP (2007). Location, location: using functional magnetic resonance imaging to pinpoint brain differences relevant to stimulant use. Addiction 102(Suppl 1): 33-43.

Becker B, Wagner D, Koester P, Bender K, Kabbasch C, GouzoulisMayfrank E et al (2012). Memory-related hippocampal functioning in ecstasy and amphetamine users: a prospective fMRI study. Psychopharmacology (Berl) 225: 923-934.

Bedi G, Phan KL, Angstadt M, de Wit H (2009). Effects of MDMA on sociability and neural response to social threat and social reward. Psychopharmacology (Berl) 207: 73-83.

Bolla KI, Eldreth DA, London ED, Kiehl KA, Mouratidis M, Contoreggi C et al (2003). Orbitofrontal cortex dysfunction in abstinent cocaine abusers performing a decision-making task. Neuroimage 19: 1085-1094.

Bolla KI, Eldreth DA, Matochik JA, Cadet JL (2005). Neural substrates of faulty decision-making in abstinent marijuana users. Neuroimage 26: 480-492.

Cowan RL, Lyoo IK, Sung SM, Ahn KH, Kim MJ, Hwang J et al (2003). Reduced cortical gray matter density in human MDMA (Ecstasy) users: a voxel-based morphometry study. Drug Alcohol Depend 72: 225-235.

Cservenka A, Nagel BJ (2012). Risky decision-making: an FMRI study of youth at high risk for alcoholism. Alcohol Clin Exp Res 36: 604-615.

Daumann J, Fischermann T, Heekeren K, Henke K, Thron A, Gouzoulis-Mayfrank E (2005). Memory-related hippocampal dysfunction in poly-drug ecstasy (3,4-methylenedioxymethamphetamine) users. Psychopharmacology (Berl) 180: 607-611.

Daumann J, Koester P, Becker B, Wagner D, Imperati D, Gouzoulis-Mayfrank E et al (2011). Medial prefrontal gray matter volume reductions in users of amphetamine-type stimulants revealed by combined tract-based spatial statistics and voxel-based morphometry. Neuroimage 54: 794-801.

De Win MM, Jager G, Booij J, Reneman L, Schilt T, Lavini C et al (2008). Sustained effects of ecstasy on the human brain: a prospective neuroimaging study in novel users. Brain 131(Pt 11): 2936-2945.

Ersche KD, Fletcher PC, Lewis SJ, Clark L, Stocks-Gee G, London $M$ et al (2005). Abnormal frontal activations related to decisionmaking in current and former amphetamine and opiate dependent individuals. Psychopharmacology (Berl) 180: 612-623.

Figee M, Vink M, de Geus F, Vulink N, Veltman DJ, Westenberg H et al (2011). Dysfunctional reward circuitry in obsessivecompulsive disorder. Biol Psychiatry 69: 867-874.

Gallinat J, Meisenzahl E, Jacobsen LK, Kalus P, Bierbrauer J, Kienast T et al (2006). Smoking and structural brain deficits: a volumetric MR investigation. Eur J Neurosci 24: 1744-1750.

Hanson KL, Luciana M, Sullwold K (2008). Reward-related decision-making deficits and elevated impulsivity among MDMA and other drug users. Drug Alcohol Depend 96: 99-110.

Hutton C, Draganski B, Ashburner J, Weiskopf N (2009). A comparison between voxel-based cortical thickness and voxelbased morphometry in normal aging. Neuroimage 48: 371-380.

Jenkinson M, Bannister P, Brady M, Smith S (2002). Improved optimization for the robust and accurate linear registration and motion correction of brain images. Neuroimage 17: 825-841.
Jenkinson M, Smith S (2001). A global optimisation method for robust affine registration of brain images. Med Image Anal 5: $143-156$.

Kalivas PW, Volkow ND (2005). The neural basis of addiction: a pathology of motivation and choice. Am J Psychiatry 162: 1403-1413.

Kish SJ, Lerch J, Furukawa Y, Tong J et al (2010). Decreased cerebral cortical serotonin transporter binding in ecstasy users: a positron emission tomography/((11)C)DASB and structural brain imaging study. Brain 133(Pt 6): 1779-1797.

Knutson B, Adams CM, Fong GW, Hommer D (2001). Anticipation of increasing monetary reward selectively recruits nucleus accumbens. J Neurosci 21: RC159.

Koester P, Tittgemeyer M, Wagner D, Becker B, GouzoulisMayfrank E, Daumann J (2012). Cortical thinning in amphetamine-type stimulant users. Neuroscience 221: 182-192.

Koob GF, Simon EJ (2009). The neurobiology of addiction: where we have been and where we are going. J Drug Issues 39: 115-132.

Kuhn S, Schubert F, Gallinat J (2010). Reduced thickness of medial orbitofrontal cortex in smokers. Biol Psychiatry 68: 1061-1065.

Lawyer G, Bjerkan PS, Hammarberg A, Jayaram-Lindstrom N, Franck J, Agartz I (2010). Amphetamine dependence and comorbid alcohol abuse: associations to brain cortical thickness. BMC Pharmacol 10: 5.

Mechtcheriakov S, Brenneis C, Egger K, Koppelstaetter F, Schocke M, Marksteiner J (2007). A widespread distinct pattern of cerebral atrophy in patients with alcohol addiction revealed by voxel-based morphometry. J Neurol Neurosurg Psychiatry 78: 610-614.

Moeller FG, Steinberg JL, Lane SD, Buzby M, Swann AC, Hasan KM et al (2007). Diffusion tensor imaging in MDMA users and controls: association with decision making. Am J Drug Alcohol Abuse 33: 777-789.

Murphy PN, Bruno R, Ryland I, Wareing M, Fisk JE, Montgomery $C$ et al (2012). The effects of ecstasy' (MDMA) on visuospatial memory performance: findings from a systematic review with meta-analyses. Hum Psychopharmacol 27: 113-138.

Paulus MP, Hozack N, Frank L, Brown GG, Schuckit MA (2003). Decision making by methamphetamine-dependent subjects is associated with error-rate-independent decrease in prefrontal and parietal activation. Biol Psychiatry 53: 65-74.

Paulus MP, Tapert SF, Schuckit MA (2005). Neural activation patterns of methamphetamine-dependent subjects during decision making predict relapse. Arch Gen Psychiatry 62: 761-768.

Quednow BB, Kuhn KU, Hoppe C, Westheide J, Maier W, Daum I et al (2007). Elevated impulsivity and impaired decision-making cognition in heavy users of MDMA ('Ecstasy'). Psychopharmacology (Berl) 189: 517-530.

Roberts GM, Garavan H (2010). Evidence of increased activation underlying cognitive control in ecstasy and cannabis users. Neuroimage 52: 429-435.

Roesch MR, Olson CR (2004). Neuronal activity related to reward value and motivation in primate frontal cortex. Science 304: 307-310.

Rogers RD, Everitt BJ, Baldacchino A, Blackshaw AJ, Swainson R, Wynne $\mathrm{K}$ et al (1999). Dissociable deficits in the decisionmaking cognition of chronic amphetamine abusers, opiate abusers, patients with focal damage to prefrontal cortex, and tryptophan-depleted normal volunteers: evidence for monoaminergic mechanisms. Neuropsychopharmacology 20: 322-339.

Rogers RD, Ramnani N, Mackay C, Wilson JL, Jezzard P, Carter CS et al (2004). Distinct portions of anterior cingulate cortex and medial prefrontal cortex are activated by reward processing in separable phases of decision-making cognition. Biol Psychiatry 55: 594-602.

Rogers RD, Tunbridge EM, Bhagwagar Z, Drevets WC, Jezzard P, Carter CS et al (2003). Tryptophan depletion alters the decisionmaking of healthy volunteers through altered processing of reward cues. Neuropsychopharmacology 28: 153-162. 
Rolls ET (2004). The functions of the orbitofrontal cortex. Brain Cogn 55: 11-29.

Schilt T, Goudriaan AE, Koeter MW, van den Brink W, Schmand B (2009). Decision making as a predictor of first ecstasy use: a prospective study. Psychopharmacology (Berl) 203: 519-527.

Schoenbaum G, Roesch MR, Stalnaker TA (2006). Orbitofrontal cortex, decision-making and drug addiction. Trends Neurosci 29: $116-124$.

Schoenbaum G, Shaham Y (2008). The role of orbitofrontal cortex in drug addiction: a review of preclinical studies. Biol Psychiatry 63: 256-262.

Smerdon MJ, Francis AJ (2011). Reward sensitivity and outcome expectancies as predictors of ecstasy use in young adults. Addict Behav 36: 1337-1340.

Smith SM (2002). Fast robust automated brain extraction. Hum Brain Mapp 17: 143-155.

Smith SM, Jenkinson M, Woolrich MW, Beckmann CF, Behrens TE, Johansen-Berg $\mathrm{H}$ et al (2004). Advances in functional and structural MR image analysis and implementation as FSL. Neuroimage 23(Suppl 1): S208-S219.

Studer B, Apergis-Schoute AM, Robbins TW, Clark L (2012). What are the odds? The neural correlates of active choice during gambling. Front Neurosci 6: 46.
Tobler PN, Christopoulos GI, O’Doherty JP, Dolan RJ, Schultz W (2009). Risk-dependent reward value signal in human prefrontal cortex. Proc Natl Acad Sci USA 106: 7185-7190.

UNODC (2011): 2011 Global ATS Assessment. United Nations Publication http://www.unodc.org/documents/ATS/ATS_Globa1_Assessment_2011.pdf.

van Hell HH, Vink M, Ossewaarde L, Jager G, Kahn RS, Ramsey NF (2010). Chronic effects of cannabis use on the human reward system: an fMRI study. Eur Neuropsychopharmacol 20: 153-163.

Volkow ND, Fowler JS, Wang GJ, Baler R, Telang F (2009). Imaging dopamine's role in drug abuse and addiction. Neuropharmacology 56(Suppl 1): 3-8.

Wagner D, Becker B, Koester P, Gouzoulis-Mayfrank E, Daumann J (2012). A prospective study of learning, memory, and executive function in new MDMA users. Addiction 108: 136-145.

Woolrich MW, Jbabdi S, Patenaude B, Chappell M, Makni S, Behrens T et al (2009). Bayesian analysis of neuroimaging data in FSL. Neuroimage 45(Suppl 1): S173-S186.

Woolrich MW, Ripley BD, Brady M, Smith SM (2001). Temporal autocorrelation in univariate linear modeling of FMRI data. Neuroimage 14: 1370-1386.

Worsley KJ, Evans AC, Marrett S, Neelin P (1992). A threedimensional statistical analysis for CBF activation studies in human brain. J Cereb Blood Flow Metab 12: 900-918. 